Article

\title{
The Influence of Vertical Vibration on Nanoscale Friction: A Molecular Dynamics Simulation Study
}

\author{
Yang Cheng ${ }^{1}$, Pengzhe Zhu ${ }^{2}$ and Rui $\mathrm{Li}^{1, * \text { (ID) }}$ \\ 1 School of Mechanical Engineering, University of Science and Technology Beijing, Beijing 100083, China; \\ chengyang911229@163.com \\ 2 School of Mechanical, Electronic and Control Engineering, Beijing Jiaotong University, Beijing 100044, China; \\ pzzhu@bjtu.edu.cn \\ * Correspondence: lirui@ustb.edu.cn
}

Received: 15 January 2018; Accepted: 7 March 2018; Published: 9 March 2018

\begin{abstract}
The influence of vibration on friction at the nanoscale was studied via molecular dynamics (MD) simulations. The results show that average friction increases in a high-frequency range. This can be attributed to the vibration of the tip following vibration excitation, which results in peaks of repulsive interaction between tip and substrate and leads to higher friction. However, when the frequency is lower than a certain value, friction decreases. This is because vibration excitation results not in an obvious vibration of the tip but in a slightly larger interface distance, which leads to a decrease in friction.
\end{abstract}

Keywords: nanoscale friction; vertical vibration; molecular dynamics

\section{Introduction}

Friction and adhesion play important roles in nano- and micro-electro mechanical systems (NEMS and MEMS) and nanosensors owing to surface and interface effects. Friction management in these systems is critical for their performance and energy saving. Many researchers have made great efforts. For example, carbon nanomaterials, which have great potential in NEMS, show the ability to tune friction by surface treatment [1-4], interface structure morphology [5-7], and the environmental factors such as temperature [8] and humidity [9]. However, these methods work only under certain situations and a more general method that can tune friction at the nanoscale is still lacking.

The research in friction at the macroscale demonstrates that the external electric field, the magnetic field, and vibration are effective in tuning friction. Moreover, nanoscale friction can also be altered mechanically [10], which may shed light on the tuning of nanoscale friction and lead to more reliable nanoscale devices. Gao et al. [11] indicated that friction in thin-film boundary lubricated junctions can be reduced by coupling small amplitude (of the order of $0.1 \mathrm{~nm}$ ) directional mechanical oscillations. Heuberger et al. [12] confirmed this conclusion with a surface forces apparatus experiment. Tshiprut, Urbakh et al. [13,14], and Ma [15] demonstrated that friction can be tuned and reduced by controlling the frequency and amplitude of the imposed substrate lateral excitations. The reason given was that the diffusion coefficient in nanoscale-confined regions can be greatly enhanced by mechanical oscillations. Capozza et al. [16,17] studied the dynamics of particles confined between a horizontally driven top plate and a vertically oscillating bottom plate. The results showed that tiny vibrations, when applied in a suitable range of frequencies, may suppress the high dissipative stick-slip dynamics and drastically reduce the lateral friction force. Socoliuc et al. [18] demonstrated that variations in interaction energy reduce friction below 10 piconewtons in a finite range of excitation and load by exciting the mechanical resonances of the sliding system perpendicular to the contact plane, without any noticeable wear. Guo et al. [19] discussed the control of vibration to reduce friction based on a 1D model imitating the 
friction force microscope (FFM) tip moving on a substrate. Fajardo et al. [20] indicated that, under out-of-plane and in-plane contact vibrations, average friction forces were both significantly lowered in a frequency range determined by the "washboard" frequency of the stick-slip motion and the viscous damping accompanying the tip motion. However, Roth et al. [21] studied the influence of lateral vibrations on the stick-slip motion by atomic force microscopy (AFM) measurements on an $\mathrm{NaCl}(001)$ surface. Their results showed the peak values of the lateral force and the total energy losses increased with the excitation amplitude, which implied that the vibration of the tip may increase friction under some situations.

Above all, it is clear that mechanical vibration of suitable frequencies and amplitudes is an effective way to suppress nanoscale friction. However, why vibration affects friction under vibration excitation needs more exploration. In this paper, the influence of out-of-plane vibration in a range of frequencies is investigated via molecular dynamics (MD) simulation. We find that friction reduces in a lower frequency range but increases in a higher frequency range. The reason why vibration causes opposite influence on friction under different frequencies is discussed.

\section{Simulation Model}

MD simulations are discussed in this paper. Figure 1a,b illustrate a simplified AFM model and an MD model, respectively. As shown in Figure 1b, a diamond tip slides on the two layers of graphene under a vertical vibration. There is a virtual atom connected to the diamond tip vertically through a harmonic spring with a stiffness $\mathrm{k}=40 \mathrm{~N} / \mathrm{m}$. The sinusoidal oscillation vibration of virtual atom in the $\mathrm{Z}$ direction is the vibration excitation, and the vibration of the diamond tip is used to simulate the vibration of the AFM cantilever and the tip. The resonance frequency of the tip is $319 \mathrm{GHz}$, which is calculated by $T=(1 / 2 \pi) \sqrt{\frac{\mathrm{k}}{\mathrm{m}}}$ [22]. Here, $\mathrm{k}$ is the spring stiffness, and $\mathrm{m}$ is the mass of the diamond tip. A virtual atom support in the $\mathrm{X}$ direction is connected to the tip by a harmonic spring with a stiffness $\mathrm{k}=40 \mathrm{~N} / \mathrm{m}$, which is set to be same as the stiffness of the vertical spring [23]. The support moves at constant speed to drag the tip to slide against the substrate. Meanwhile, the virtual atom in the $\mathrm{Z}$ direction moves at the same speed as the diamond tip in the $\mathrm{X}$ direction.

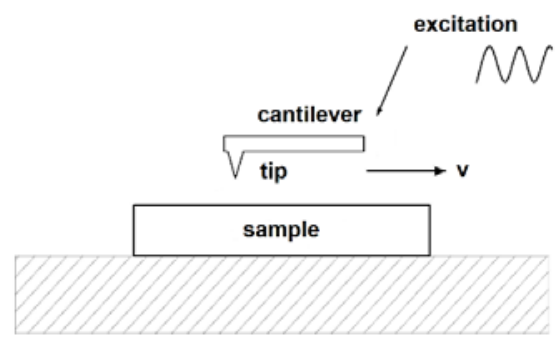

(a)

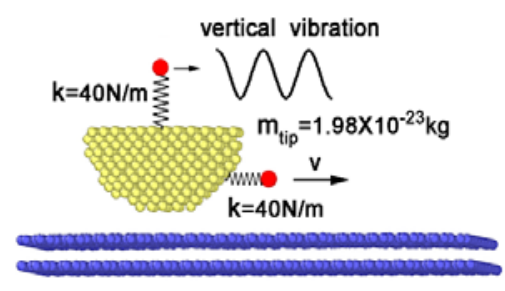

(b)

Figure 1. (a) A simplified schematic of a dynamic atomic force microscope, in which an excitation to the shaking piezo results in the tip's oscillation. (b) Molecular dynamics (MD) model: the diamond tip on two layers of graphene.

In the MD model, the diamond tip's radius is $1.1 \mathrm{~nm}$. The $X$ and $Y$ dimensions of the substrate are 3.68 and $6.39 \mathrm{~nm}$, respectively. The bottom graphene layer is fixed. To constrain translational movement of the upper graphene layer during tip sliding, the atoms at the ends of the upper graphene layer along the $\mathrm{X}$ and $\mathrm{Y}$ directions are also fixed. The remaining atoms in the upper graphene layer thermally vibrate. The diamond tip is treated as a rigid body. In all simulations, except where noted, periodic boundary conditions are used along the $X$ and $Y$ directions. The inter-atomic interactions for the diamond tip and the graphene are described by the second reactive empirical bond-order (REBO) potential [24], which has been successfully used to study the mechanical and tribological properties of carbon materials. Classic 12-6 Lennard-Jones potential is applied to describe the 
non-bonded interaction between the diamond tip and the graphene. The parameters can be found in the literature [25]. A canonical ensemble (NVT ensemble) is employed. The system's constant temperature of $100 \mathrm{~K}$ is kept by applying the Langevin thermostat. The Velocity-Verlet algorithm is used with a time step of $1 \mathrm{fs}$.

The simulation process is as follows: first, the structures of the simulation systems are optimized through energy minimization in a conjugate gradient method. Then, a relaxation is processed for $10 \mathrm{ps}$ to achieve equilibrium. Next, an external normal load of $10.56 \mathrm{nN}$ is applied to the diamond tip. The system is further relaxed under load. After relaxation, the virtual atom support in the $X$ direction, which is coupled to the diamond tip in the MD model, slides at a constant speed of $1 \mathrm{~m} / \mathrm{s}$ in the $\mathrm{X}$ direction.

\section{Results and Discussion}

The oscillation amplitude and frequency of the diamond tip are two important parameters in vibration. Since the virtual atom and diamond tip are connected via a harmonic spring, and the vibration of the diamond tip is caused by the vibration of the virtual atom, so their frequencies are the same. The resonance frequency of the diamond tip is $319 \mathrm{GHz}$. Reinstaedtler et al. [26] pointed out that cantilever torsional vibration resonances in contact with a sample surface as a function of excitation amplitude were resembled a Lorentz curve. Considering that the resonance of a diamond tip with the excitation may be similar, several frequencies $330 \mathrm{GHz}, 285 \mathrm{GHz}$, and $250 \mathrm{~Hz}$ that are near resonance frequency are calculated. The higher frequencies-3.330 $\times 10^{3} \mathrm{GHz}$ and $3.333 \times 10^{4} \mathrm{GHz}$-and lower frequencies-100 GHz and $10 \mathrm{GHz}$ - are also considered. Figure 2 shows the relationship between the amplitude of the diamond tip and the excitation. The vibration amplitude in each case is the average of several periods. The results show that the shape of the curves is similar to that of a Lorentz curve. The vibration amplitude of the diamond tip increases as the virtual atom's vibration amplitude increases. The peak of the vibration amplitude of the tip appears near resonance frequency owing to the resonance. When the vibration frequency is far away from the resonance frequency, such as $3.333 \times 10^{4} \mathrm{GHz}$, the diamond tip does not vibrate.

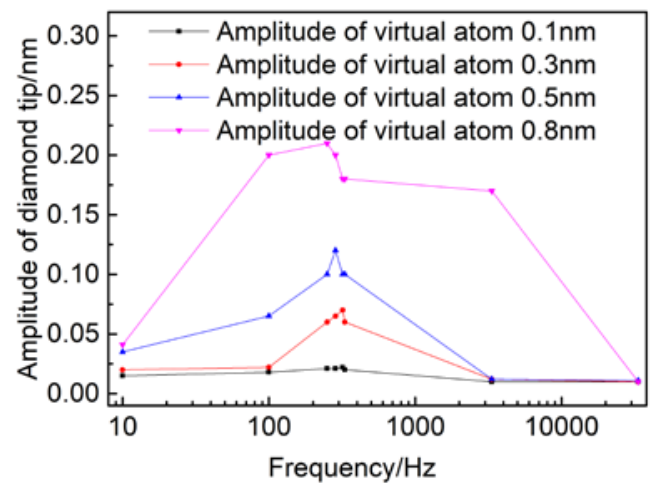

Figure 2. Vibration amplitude of the diamond tip with vibration amplitude of the virtual atom. Vibration frequencies in 8 cases are 10, 100, 250, 285, 319, 330, $3.33 \times 10^{3}, 3.333 \times 10^{4} \mathrm{GHz}$, respectively.

The average friction forces under frequencies from $10 \mathrm{MHz}$ to $3.333 \times 10^{4} \mathrm{GHz}$ were calculated. The vibration frequencies are divided into two frequency ranges. In the first range, the vibration frequencies are the same as in Figure 2, which varies from 10 to $3.333 \times 10^{4} \mathrm{GHz}$. The second range includes 7 cases with vibration frequencies $10^{3}$ times smaller than the frequencies in the first range. The frequencies are $3.330 \times 10^{3}, 330,319,285,250,100$, and $10 \mathrm{MHz}$, respectively, which ranges from $10 \mathrm{MHz}$ to $3.33 \mathrm{GHz}$, and the highest frequency in this range is about $10^{2}$ times lower than the resonance frequency. The average friction forces of the diamond tip within a higher vibration frequency range are shown in Figure $3 a, b$, in which vibration amplitude of the virtual atom is 0.1 and 
$0.5 \mathrm{~nm}$, respectively. The vibration amplitude of the tip is 0.01 and $0.1 \mathrm{~nm}$ at resonance frequency. The results show that the average friction forces are obviously larger than the value $0.057 \mathrm{nN}$ in the case without vibration except for the cases under vibration frequencies of $3.333 \times 10^{4}$ and $10 \mathrm{GHz}$ and a vibration excitation amplitude of $0.1 \mathrm{~nm}$. In these two cases, friction forces are close to that in the case without vibration. Compared to the result in Figure 2, we find that the diamond tip barely vibrates in these two cases. When the vibration amplitude of the virtual atom becomes larger, the average friction force increases more obviously, as shown in Figure 3b. However, in the lower frequency range from $10 \mathrm{MHz}$ to $3.33 \mathrm{GHz}$, the average friction forces of the diamond tip obviously reduce, as shown in Figure $4 \mathrm{a}, \mathrm{b}$, in which the amplitude of the virtual atom is also 0.1 and $0.5 \mathrm{~nm}$, respectively. Under frequencies of $319 \mathrm{MHz}, 285 \mathrm{~Hz}$, and $250 \mathrm{~Hz}$, friction forces of the diamond tip reach the lowest. Fajardo [20] pointed out that, when $v_{s} / a \leq f \leq f_{p} / Y$, friction decreases under external vibration, where $f$ is the vibration frequency, $v_{s}$ is the support velocity, $a$ is the spatial periodicity, and $f_{p}$ is the resonance frequency. $Y=\gamma /\left(2 \pi f_{p}\right)$, where $\gamma$ is a microscopic friction coefficient describing the coupling with the phonon and the possible electron oscillations in the substrate. They calculated the case with $Y=1$. Our simulation results show that, when vibration frequency is lower than $10 \mathrm{GHz}$ and the amplitude of vibration excitation is $0.1 \mathrm{~nm}$, friction is suppressed, which agrees with the formula proposed by Fajardo, except that $Y$ is much smaller than 1 . However, our results also show that friction increases when the vibration frequency is between 10 and $3.330 \times 10^{3} \mathrm{GHz}$.
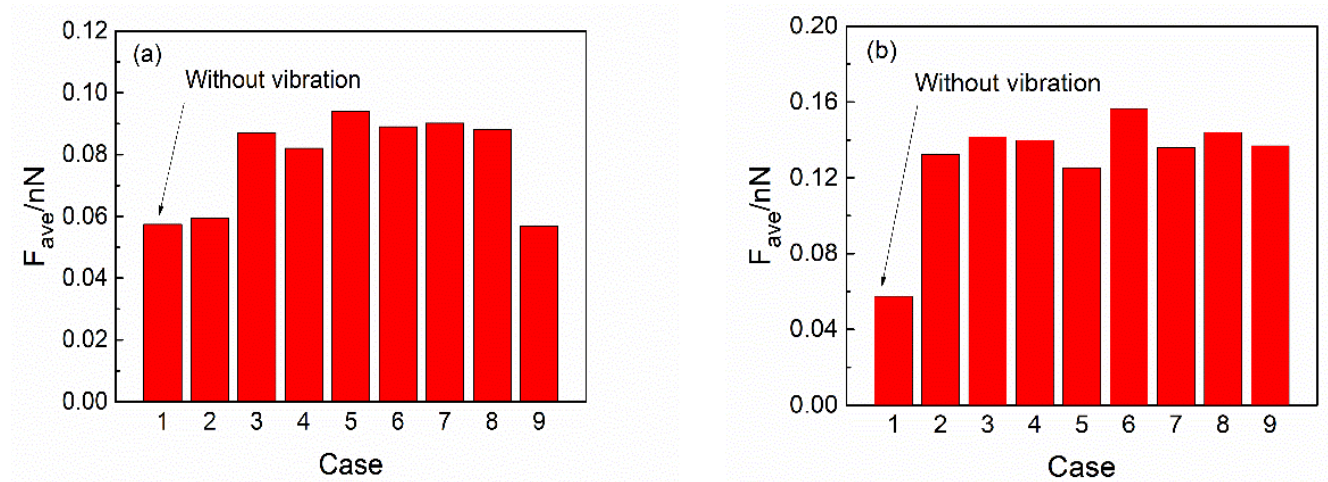

Figure 3. The average friction in a higher vibration frequency range. (a) Vibration amplitude of the virtual atom is $0.1 \mathrm{~nm}$. (b) Vibration amplitude of the virtual atom is $0.5 \mathrm{~nm}$. Vibration frequencies in 2-9 cases are $3.333 \times 10^{4}, 3.330 \times 10^{3}, 330,319,285,250,100$, and $10 \mathrm{GHz}$, respectively.
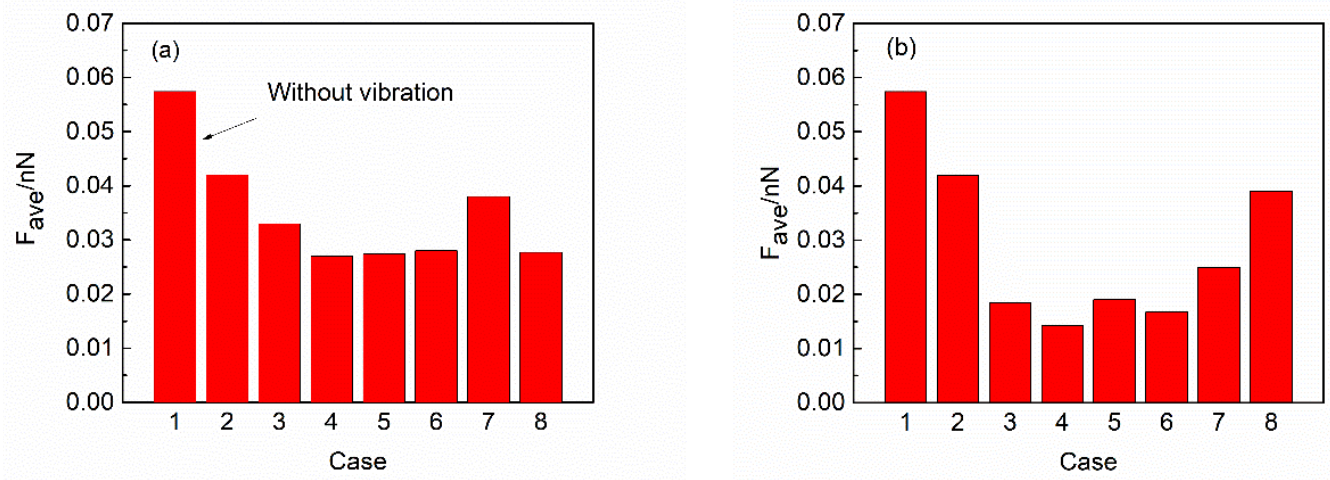

Figure 4. The average friction in lower vibration frequency range. (a) Vibration amplitude of the virtual atom is $0.1 \mathrm{~nm}$. (b) Vibration amplitude of the virtual atom is $0.5 \mathrm{~nm}$. Vibration frequencies in 2-8 cases are $3.330 \times 10^{3}, 330,319,285,250,100$, and $10 \mathrm{MHz}$, respectively.

In order to explain how and why vibration influences friction in the opposite way in two frequency ranges, first, the cases with virtual atom vibration amplitude $0.1 \mathrm{~nm}$ were analyzed. As mentioned 
above, the diamond tip does not vibrate in cases under frequencies of $3.333 \times 10^{4}$ and $10 \mathrm{GHz}$, so friction is close to the case without vibration. It can be concluded that the vibration of the diamond tip plays an important role in increasing friction. We investigated the interface distance and the normal force of the diamond tip with a virtual atom vibration amplitude of $0.5 \mathrm{~nm}$ when frequency is $100 \mathrm{GHz}$ and $100 \mathrm{MHz}$, as shown as Figure 5. In the case without vibration, the interface distance is about $1.36 \mathrm{~nm}$. When vibration frequency is $100 \mathrm{GHz}$, the interface distance in the $\mathrm{Z}$ direction varies from 1.32 to $1.45 \mathrm{~nm}$. When interface distance is smallest, the corresponding peak occurs in a lateral force curve in Figure 5b, which is about three times larger than without vibration. Because greater normal force leads to higher friction, average friction finally increases under vertical vibration. In the case of a frequency of $100 \mathrm{MHz}$, because the frequency is far from the resonance frequency of the diamond tip, the tip does not follow the sinusoidal vibration of the virtual atom. Interface distance remains at about $1.38 \mathrm{~nm}$, which is slightly larger than that in the case without vibration, as shown in Figure 5 a. The normal force on the diamond tip is obviously lower, as shown in Figure 5b, which leads to a decrease in friction. The larger interface distance under a frequency of $100 \mathrm{Mz}$ could be explained by dynamics stiffness of the spring, which connects the virtual atom and the diamond tip. According to the dynamics stiffness analysis of the single freedom spring [27], the dynamics stiffness of the spring in general will become larger as frequency increases, except for frequencies lower than about 1.3 times the resonance frequency, a range in which the dynamics stiffness of the spring becomes lower. Compared to the model in this paper, the dynamics stiffness of the spring under a $100 \mathrm{MHz}$ frequency is lower than the static stiffness. Therefore, under certain loads, the length of the spring under $100 \mathrm{Mz}$ is shorter than the case without vibration, so interface distance slightly increases. The dynamics model can also explain the cases under high frequencies, in which the diamond tip barely vibrates too. However, because of the similar or larger dynamics stiffness compared with the static stiffness, the interface distance will remain the same or decrease. Therefore, in such cases, friction, compared to cases without vibration, is similar or larger. Additionally, it should be mentioned that Li et al. [28] indicated that, in conventional MD simulation, MD results at high speeds were not in the thermally activated regime. Considering the low speed of the AFM tip in the experiment, the tip spends more time above each graphene atom and is able to escape when the potential is closer to a lower point, which may suppress the increase in friction under higher frequency.
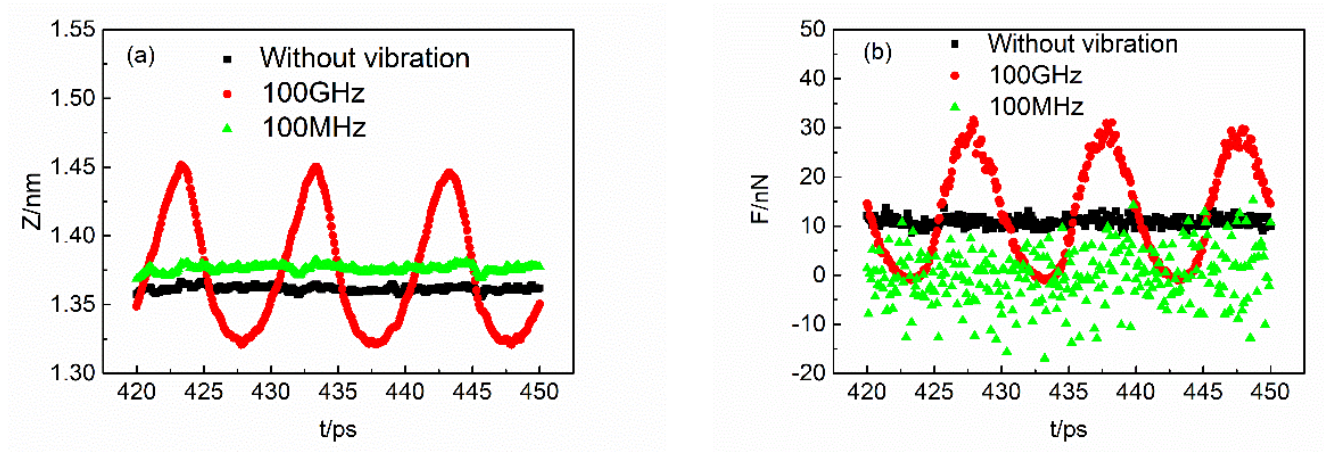

Figure 5. Interface distance and normal force of the diamond tip in three cases: the case without vibration and the cases under frequencies of $100 \mathrm{GHz}$ and $100 \mathrm{MHz}$. Vibration amplitude of the virtual atom is $0.5 \mathrm{~nm}$. (a) Interface distance with time. (b) Normal force in the $\mathrm{Z}$ direction with time.

\section{Conclusions}

In this paper, the influence of vibration on friction at the nanoscale in a range of frequencies was investigated via MD simulations. The results show that average friction increases when frequency is between 10 and $3.333 \times 10^{4} \mathrm{GHz}$. The reason for this is that diamond tips vibrate following sinusoidal vibration of the virtual atom, which results in peaks of repulsive interaction between tip and substrate and leads to higher friction. Considering the low speed of the AFM tip in the experiment, the tip is 
able to escape when the potential is closer to a lower point, which may suppress the increase in friction under higher frequency. When vibration frequency is lower than $10 \mathrm{GHz}$, vibration of the virtual atom slightly increases interface distance and results in a reduction in normal force of the diamond tip, which decreases friction force on the tip.

Acknowledgments: The work was supported by the National Natural Science Foundation of China (No. 51475039 and No. 51405337).

Author Contributions: Yang Cheng conducted the simulation and wrote the paper. Rui Li designed the simulation cases, discussed the results with Yang Cheng and revised the paper. Pengzhe Zhu had helpful discussion with Rui Li and help revise the paper.

Conflicts of Interest: The authors declare no conflict of interest.

\section{References}

1. Berman, D.; Erdemir, A.; Zinovev, A.V.; Sumant, A.V. Nanoscale friction properties of graphene and graphene oxide. Diam. Relat. Mater. 2015, 54, 91-96. [CrossRef]

2. Li, Q.Y.; Liu, X.Z.; Kim, S.P.; Shenoy, V.B.; Sheehan, P.E.; Robinson, J.T.; Carpick, R.W. Fluorination of Graphene Enhances Friction Due to Increased Corrugation. Nano Lett. 2014, 14, 5212-5217. [CrossRef] [PubMed]

3. Ko, J.H.; Kwon, S.; Byun, I.S.; Choi, J.S.; Park, B.H.; Kim, Y.H.; Park, J.Y. Nanotribological Properties of Fluorinated, Hydrogenated, and Oxidized Graphenes. Tribol. Lett. 2013, 50, 137-144. [CrossRef]

4. Men, X.H.; Zhang, Z.Z.; Yang, J.; Zhu, X.T.; Wang, K.; Jiang, W. Effect of Different Functional Carbon Nanotubes on the Tribological Behaviors of Poly (Furfuryl Alcohol)-Derived Carbon Nanocomposites. Tribol. Trans. 2011, 54, 265-274. [CrossRef]

5. Zhou, M.; Liu, K.; Wan, J.; Li, X.; Jiang, K.L.; Zeng, H.B.; Zhang, X.J.; Meng, Y.G.; Wen, S.Z.; Zhu, H.W.; Tian, Y. Anisotropic interfacial friction of inclined multiwall carbon nanotube array surface. Carbon 2012, 50, 5372-5379. [CrossRef]

6. Dickrell, P.L.; Sinnott, S.B.; Hahn, D.W.; Raravikar, N.R.; Schadler, L.S.; Ajayan, P.M.; Sawyer, W.G. Frictional anisotropy of oriented carbon nanotube surfaces. Tribol. Lett. 2005, 18, 59-62. [CrossRef]

7. Lucas, M.; Zhang, X.H.; Palaci, I.; Klinke, C.; Tosatti, E.; Riedo, E. Hindered rolling and friction anisotropy in supported carbon nanotubes. Nat. Mater. 2009, 8, 876-881. [CrossRef] [PubMed]

8. Greiner, C.; Felts, J.R.; Dai, Z.T.; King, W.P.; Carpick, R.W. Controlling Nanoscale Friction through the Competition between Capillary Adsorption and Thermally Activated Sliding. ACS Nano 2012, 6, 4305-4313. [CrossRef] [PubMed]

9. Tsai, P.C.; Jeng, Y.R.; Mao, C.P.; Wu, K.T.; Hong, F.C.N. Effects of surface morphology, size effect and wettability on interfacial adhesion of carbon nanotube arrays. Thin Solid Films 2013, 545, 401-407. [CrossRef]

10. Carpick, R.W. Controlling Friction. Science 2006, 313, 184-185. [CrossRef] [PubMed]

11. Gao, J.; Luedtke, W.D.; Landman, U. Friction Control in Thin-Film Lubrication. J. Phys. Chem. B 1998, 102, 5033-5037. [CrossRef]

12. Heuberger, M.; Drummond, C.; Israelachvili, J. Coupling of Normal and Transverse Motions during Frictional Sliding. J. Phys. Chem. B 1998, 102, 5038-5041. [CrossRef]

13. Tshiprut, Z.; Filippov, A.E.; Urbakh, M. Tuning Diffusion and Friction in Microscopic Contacts By Mechanical Excitations. Phys. Rev. Lett. 2005, 95, 016101. [CrossRef] [PubMed]

14. Guerra, R.; Vanossi, A.; Urbakh, M. Controlling microscopic friction through mechanical oscillations. Phys. Rev. E 2008, 78, 036110. [CrossRef] [PubMed]

15. Ma, M.; Sokolov, I.M.; Wang, W.; Filippov, A.E.; Zheng, Q.; Urbakh, M. Diffusion through Bifurcations in Oscillating Nano- and Microscale Contacts: Fundamentals and Applications. Phys. Rev. X 2015, 5, 031020. [CrossRef]

16. Capozza, R.; Vanossi, A.; Vezzani, A.; Zapperi, S. Suppression of Friction by Mechanical Vibrations. Phys. Rev. Lett. 2009, 103, 085502. [CrossRef] [PubMed]

17. Capozza, R.; Vanossi, A.; Vezzani, A.; Zapperi, S. Triggering Frictional Slip by Mechanical Vibrations. Tribol. Lett. 2012, 48, 95-102. [CrossRef] 
18. Socoliuc, A.; Gnecco, E.; Maier, S.; Pfeiffer, O.; Baratoff, A.; Bennewitz, R.; Meyer, E. Atomic-scale control of friction by actuation of nanometer-sized contacts. Science 2006, 313, 207-210. [CrossRef] [PubMed]

19. Guo, Y.; Wang, Z.; Qu, Z.; Braiman, Y. Atomic-scale friction control by vibration using friction force microscope. Control Eng. Pract. 2011, 19, 1387-1397. [CrossRef]

20. Fajardo, O.Y.; Gnecco, E.; Mazo, J.J. Out-of-plane and in-plane actuation effects on atomic-scale friction. Phys. Rev. B 2014, 89, 075423. [CrossRef]

21. Roth, R.; Fajardo, O.Y.; Mazo, J.J.; Meyer, E.; Gnecco, E. Lateral vibration effects in atomic-scale friction. Appl. Phys. Lett. 2014, 104, 083103. [CrossRef]

22. Hu, X.; Egberts, P.; Dong, Y.; Martini, A. Molecular dynamics simulation of amplitude modulation atomic force microscopy. Nanotechnology 2015, 26, 235705. [CrossRef] [PubMed]

23. Shimizu, J.; Eda, H.; Yoritsune, M.; Ohmura, E. Molecular dynamics simulation of friction on the atomic scale. Nanotechnology 1998, 9, 118-123. [CrossRef]

24. Stuart, S.J.; Tutein, A.B.; Harrison, J.A. A reactive potential for hydrocarbons with intermolecular interactions. J. Chem. Phys. 2000, 112, 6472-6486. [CrossRef]

25. Neek-Amal, M.; Peeters, F.M. Nanoindentation of a circular sheet of bilayer graphene. Phys. Rev. B 2010, 81 , 235421. [CrossRef]

26. Reinstädtler, M.; Rabe, U.; Goldade, A.; Bhushan, B.; Arnold, W. Investigating ultra-thin lubricant layers using resonant friction force microscopy. Tribol. Int. 2005, 38, 533-541. [CrossRef]

27. Liu, L.; Zhang, W.H. Frequency variety analysis and equivalent algorithm of metal spring stiffness. J. Traffic Transp. Eng. 2007, 7, 24-27.

28. Li, Q.Y.; Dong, Y.L.; Perez, D.; Martini, A.; Carpick, R.W. Speed Dependence of Atomic Stick-Slip Friction in Optimally Matched Experiments and Molecular Dynamics Simulations. Phys. Rev. Lett. 2011, 106, 126101. [CrossRef] [PubMed]

(C) 2018 by the authors. Licensee MDPI, Basel, Switzerland. This article is an open access article distributed under the terms and conditions of the Creative Commons Attribution (CC BY) license (http:/ / creativecommons.org/licenses/by/4.0/). 